\section{Sex differences in adherence to guidelines in aspirin prescription in a population of low-risk cardiovascular patients}

\author{
Anna Vittoria Mattioli, Antonio Manenti and Alberto Farinetti
}

European Journal of Preventive Cardiology $0(00) \mathrm{I}-2$

(C) The European Society of Cardiology 2018

Reprints and permissions: sagepub.co.uk/journalsPermissions.nav DOI: 10.1 I $77 / 20474873$ | 8758433 journals.sagepub.com/home/ejpc

(S)AGE
Dear Editor,

We have read with great interest the paper Age and gender differences in medical adherence after myocardial infarction: Women do not receive optimal treatmentThe Netherlands claims database by Eindhoven and coworkers $^{1}$ and we found it of importance with a view to clinical prevention. The authors assess medical adherence during 1 year following myocardial infarction, stratifying per type of infarct, age and sex. They concluded that age and sex differences existed in medical adherence after myocardial infarction. Medical adherence was lower in women, young patients and elderly patients, specifically in non-ST-elevation myocardial infarction (NSTEMI) patients.

With reference to the findings reported in the paper, we would like to make the following contribution to the discussion. Aspirin is widely used in low risk patients with cardiovascular disease to prevent thrombosis, despite the increasing risk of bleeding. We evaluated the adherence to guidelines in aspirin prescription in 2095 patients (960 men (45.8\%) and 1135 women (54.2\%), mean age $58 \pm 12$ years) affected by minor rectal hemorrhage. The daily aspirin dosage ranged from 80 to $500 \mathrm{mg}$. We found that 220 patients were treated with aspirin $(10.9 \%)$, specifically they were 120 men $(12.5 \%)$ and 100 women $(9.4 \%),(p<0.05)$. Of these 220,205 $(75.1 \%)$ were treated with aspirin according to the guidelines for prevention of cardiovascular disease; 10 patients were treated with aspirin out of guideline indications. In addition, 683 patients (32.6\%) that needed aspirin or anti-platelet therapy due to their cardiovascular risk were not treated, despite not having contraindications or allergy. Adherence to guidelines was higher in men compared with women. The occurrence of previous small peripheral hemorrhage did not limit the use of antiplatelet therapy, on the contrary, in real life we found that is considered a limit for prescription.

Our population was characterized by a low cardiovascular risk as indicated by the Framingham score risk; however, we report a low adherence regarding drugs prescription, mainly in women.
Discontinuation of secondary prevention with aspirin has been associated with higher risk of cardiovascular events in some studies ${ }^{2,3}$ with indications of an increased risk shortly after discontinuation. Aspirin is often withdrawn because of surgery or bleeding, factors that per se may stimulate platelet aggregation and increase the risk of cardiovascular events. $4,5,6$

A high initial discontinuation rate was reported in a previous study: 1 of 5 aspirin-naïve patients did not collect the second prescription of aspirin dispensed, and the main persistence drop was during the first year after aspirin initiation. In contrast, those who picked up their second prescription had a modest discontinuation rate over time. ${ }^{7}$

We think that larger clinical trials evaluating the effects of poor adherence to therapy and the risk of cardiovascular events in women with minor hemorrhage should be conducted. It is well known that there are significant disparities between women and men in their intensity of medication use, and their likelihood of receiving guideline-based drug therapy. ${ }^{8}$ These differences indicate a need for more personalized drug management to reduced clinical events.

\section{Author contribution}

AVM and AF contributed to the conception and design of the work. AM and AF contributed to the acquisition and analysis of data for the work. AVM drafted the manuscript. AF critically revised the manuscript. All gave final approval and agree to be accountable for all aspects of work ensuring integrity and accuracy.

Surgical, Medical and Dental Department of Morphological Sciences Related to Transplant, Oncology and Regenerative Medicine University of Modena and Reggio Emilia, Italy

\section{Corresponding author:}

Anna Vittoria Mattioli, Department of Surgical, Medical and Dental Department of Morphological Sciences Related to Transplant, Oncology and Regenerative Medicine, University of Modena and Reggio Emilia, Via del Pozzo, 7I 4II00 Modena, Italy.

Email: annavittoria.mattioli@unimore.it 


\section{Declaration of conflicting interests}

The authors declared no potential conflicts of interest with respect to the research, authorship, and/or publication of this article.

\section{Funding}

The authors received no financial support for the research, authorship, and/or publication of this article.

\section{References}

1. Eindhoven DC, Hilt AD, Zwaan TC, et al. Age and gender differences in medical adherence after myocardial infarction: Women do not receive optimal treatment-The Netherlands claims database. Eur J Prev Cardiol 2018; 25: $181-189$.

2. Mattioli AV, Bonetti L, Zennaro M, et al. Acute myocardial infarction in young patients: Nutritional status and biochemical factors. Int J Cardiol 2005; 101: 185-190.

3. Rodríguez LA, Cea-Soriano L, Martín-Merino E, et al. Discontinuation of low dose aspirin and risk of myocardial infarction: Case-control study in UK primary care. BMJ 2011; 343: d4094.

4. Burger W, Chemnitius JM, Kneissl GD, et al. Low-dose aspirin for secondary cardiovascular prevention: cardiovascular risks after its perioperative withdrawal versus bleeding risks with its continuation: Review and meta-analysis. J Intern Med 2005; 257: 399-414.

5. Vinther JL, Jacobsen RK and Jørgensen T. Current European guidelines for management of cardiovascular disease: Is medical treatment in nearly half a population realistic? Eur J Prev Cardiol 2018; 25: 157-163.

6. Xing M, Shi Y, Zhu X, et al. How aspirin prevents cardiovascular diseases. Eur J Prev Cardiol. Epub ahead of print 9 Jan 2018. DOI: 10.1177/2047487317751732.

7. Sundström J, Hedberg J, Thuresson M, et al. Low-dose aspirin discontinuation and risk of cardiovascular events: A Swedish nationwide, population-based cohort study. Circulation 2017; 136: 1183-1192.

8. Manteuffel M, Williams S, Chen W, et al. Influence of patient sex and gender on medication use, adherence, and prescribing alignment with guidelines. $J$ Womens Health (Larchmt) 2014; 23: 112-119. 